\title{
PREFACE: IS HETEROCYCLIC CHEMISTRY BORING?
}

A note with this provoking title was published by the author in $1966 .{ }^{1}$ Although the question was answered in the negative, it is amusing to compare status and perspectives of heterocyclic chemistry formerly and now.

Heterocycles are inextricably woven into the processes of organic life. Beyond nucleic acids and carbohydrates; we may quote macrocyclic dyes, alkaloids, vitamins, and antibiotics as examples, Connected with the natural occurrence is the vital interest of the pharmaceutical and agrochemical industries in heterocyclic chemistry. Synthetic chemistry showed that carbocycles tolerate many other elements in variable numbers as ring members. Should pentazoles $\left(R N_{5}\right)$ or the $P_{4}$ tetrahedron still be regarded as heterocycles with all ring members coming from the same element? The substitution of carbon atoms in condensed polycyclic compounds by heterofunctions multiplies the numbers of conceivable structures. Statistical analysis recalls the notion that the number of organic compounds is infinite; a majority is heterocyclic.

The borders of the countries are neatly defined on an atlas, This is not so in the arrangement of chemical sciences into disciplines; overlap is thwarting clear divisions. Heterocyclic chemistry, in fact, pervades the plurality of the other chemical disciplines. The objection that the near omnipresence of heterocycles renders their separate treatment superfluous, is easily refuted: the modification and alteration of chemical and physical properties of molecules by heteroatoms argue for a special discipline.

The sheer volume suggests a reshaping of the title question: Is heferocyclic chemistry frightening? The lack of consistency in the many-faceted discipline was deplored by the author in 1966. Today, heterocyclic synthesis is no longer a maze. The number of general synthetic principles which allow the construction of more than one heterocyclic system, is increasing. Pericyclic reactions are especially tolerant with respect to kind and number of heteroatoms. In modern lecture courses and textbooks, retrosynthetic principles are valuable and provide an abundance of test questions.

Heterocyclic chemistry is no longer the chaotic tropical garden it used to be 50 years ago. Spectroscopic studies, mechanistic investigations, and theoretical calculations led to a deeper understanding of static and dynamic aspects of heterocycles.

Drug research in pharmaceutical industry deals to a large extent with heterocycles. Innumerable structural variants have to be synthesized in the course of optimization, before a medicine is released. Modern combinatorial chemistry enables an effortless multiplication of the number of substances for highly automated bio-tests. 
In the past, the "synthesis of compounds of potential biological interest" often occurred in grant applications. Only a new synthetic principle or a novel pharmacological activity as guiding motif can justify such projects in university research. Just making new compounds for biological testing without a specific goal is not an intellectual challenge; it has even discredited heterocyclic chemistry to a certain extent.

Since our note of 1966, the International Society of Heterocyclic Chemistry was founded and represents a well-established discipline. Biannual International Congresses strengthen the feeling of solidarity among heterocyclic chemists; the first Congress was held in Albuquerque, Now Mexico, in 1967 and the sixteenth in Bozeman, Montana, in 1997. Scientists of outstanding merit are honored by the International Award of Heterocyclic Chemistry.

Two international journals serve heterocyclic chemistry in its full breadth, in addition to several more specialized periodicals. The Journal of Heterocyclic Chemistry was founded by Raymond N. Castle in 1964, and Tetsuji Kametani Initiated Heterocycles in 1973. Furthermore, the Advances of Heterocyclic Chemistry were set up by Alan R. Katritzky in 1962, and Vol. 70 (1998) was still edited by the same active scientist.

After the recent 25th anniversary, Heterocycles now commemorates its 50 th Volume. This is ample reason to celebrate; the Editor and his staff may be congratulated to a story of success. The now journal was rapidly accepted and captured an essential position in the heterocyclic literature. Heterocycles offers communications, full papers, and reviews; the columns on new natural products and total syntheses are useful, too. The high scientific reputation of the Editors - Tetsuji Kametani succeeded by Keiichiro Fukumoto - and careful reviewing have guaranteed a high standard of the journal.

A periodical based on camera-ready manuscripts should publish the contributions in record time. This is the case. However, an even influx of manuscripts remains a dream of the editorial staff; occasional "bumper crops" generate waiting times. Printed on quality paper, the make-up of Heterocycles is very attractive. A greater uniformity of the articles might be achieved by more detailed general instructions.

Not much imagination is required to predict a good future for Heterocycles. May success accompany the journal into the next millennium!

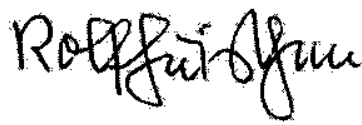

Rolf Huisgen

University of München, Germany

1. R. Huisgen, Nachrichten aus Chemie und Technik (Verlag Chemie, Weinhein), 487 (1966). 